\title{
Acute Type A Aortic Dissection during Pregnancy: Trials and Tribulations
}

\author{
Lara Rimmer ${ }^{1}$, Jessica Heyward-Chaplin ${ }^{1}$, Matthew South ${ }^{1}$, Mohamed Gouda $^{2}$, and \\ Mohamad Bashir ${ }^{3}$ \\ ${ }^{1}$ East Lancashire Hospitals NHS Trust \\ ${ }^{2}$ Mataria Teaching Hospital \\ ${ }^{3}$ Royal Blackburn Teaching Hospital
}

August 14, 2020

\begin{abstract}
Type A acute aortic dissection (TAAD) during pregnancy is a life-threatening event for both the mother and unborn baby. Pregnancy has been recognised as an independent risk factor for TAAD, postulated to be due to physiological changes that cause hyperdynamic circulation. Presentation can be atypical in many cases and further concern from clinicians of fetal radiation exposure can result in missed or delayed diagnoses. Investigation via quickest form of imaging, whether CT, MRI or transoesophageal echocardiography, should be carried out promptly due to the high risk of mortality. Surgical management of TAAD in pregnancy revolves primarily around the decision to deliver the foetus concomitantly or to perform aortic repair with the foetus in utero. This review will summarise the difficulties faced when managing TAAD in pregnancy, and important questions for future research.
\end{abstract}

\section{Key}

TAAD - Type A Acute Aortic Dissection

AAD - Acute Aortic Dissection

AD - Aortic Dissection

ASI - Aortic Size Index

BAV - Bicuspid Aortic Valve

CTD - Connective Tissue Disease

HTAD - Heritable Thoracic Aortic Disease

MFS - Marfan Syndrome

TOE - Transoesophageal Echocardiography

TTE - Transthoracic Echocardiography

TS - Turner Syndrome

vEDS - Vascular Ehlers-Danlos Syndrome

Introduction 
Type A acute aortic dissection (TAAD) in pregnancy is a rare but potentially lethal event for both mother and foetus. TAAD cases have been reported on extensively in literature from 1944, and yet remains a considerable unknown in the best ways of management ${ }^{1}$. This is due to the low incidences of cases and limited experiences in managing these women. The International Registry of Aortic Disease enrolled pregnant women from 1998 to 2019, and found only 29 cases across 17 different sites in 20 years ${ }^{2}$. Similarly, Kamel et al. performed a cohort-crossover analysis on emergency department visits across several states of the US, identifying 36 cases of AAD during pregnancy in 6566826 pregnancies; an absolute risk of 5.5 per million cases ${ }^{3}$. Regardless of the rarity of the incidence of the disease, the mortality is high enough $(21-53 \%)$ for it to be notable ${ }^{4}$. Maternal mortality rates increase by 1-3\% per hour and exceed $80 \%$ during the first month if left untreated ${ }^{5}$.

The highest incidence of TAAD is in the third trimester $(50 \%)$ and the early postpartum period $(33 \%)^{6}$. This suggests a link between the integrity of the aortic wall and the cardiocirculatory changes in late pregnancy ${ }^{7,8}$. Oestrogen and progesterone cause structural changes in the intima and media ${ }^{9}$. Increased systemic vascular resistance in pregnancy increases afterload and reduces the preload, causing endothelial injury and risk of $\mathrm{AD}^{10}$. Marfan Syndrome (MFS) and other connective tissue disorders are also important predisposing factors for aortic dissection in pregnancy ${ }^{11}$.

Many women present acutely with sudden-onset, severe chest pain and haemodynamic instability ${ }^{7,12,13}$. Although more subtle presentations can easily be mistaken for common pregnancy complaints, such as uncontrolled hypertension, gastro-oesophageal reflux and neurological deficits ${ }^{14}$. Misdiagnoses of acute myocardial infarction, pulmonary embolism, pre-eclampsia or pancreatitis can have detrimental effect to initiation of treatment ${ }^{12}$. As such, clinicians should have a low threshold of suspicion; any pregnant female with acute chest pain should be considered as possible $\mathrm{AD}$ and investigated urgently ${ }^{14}$. Any pregnant woman with aortopathy should have full imaging of her aorta as soon as possible ${ }^{15}$.

\section{Materials and Methods}

A comprehensive literature search was carried out across large databases to assimilate relevant papers regarding AAD in pregnant women.

Results

\section{Investigating TAAD in Pregnant Women}

CT aortography, MRI and transoesophageal echocardiography (TOE) have all proven useful in the diagnosis of TAAD in pregnancy. The historical gold standard for the diagnosis of TAAD is CT aortography ${ }^{16}$. In pregnancy, there is often concern raised about potential fetal harm linked with radiation exposure. This fear could potentially lead to delayed or missed diagnosis.

Varying recommendations regarding the optimum investigation strategy for these women exists across different governing bodies. The American College of Obstetricians and Gynecologists recommend that ionizing radiation through the use of radiography, CT or nuclear imaging should not be withheld from a pregnant woman if it is more readily available or thought to be necessary in addition to ultrasonography or MRI14. This is because, with few exceptions, the radiation dose is much lower than the exposure associated with fetal harm ${ }^{17,18}$. In a similar sentiment, the European Society of Cardiology recommend for haemodynamically unstable patients, the quickest form of imaging should be utilised, whether MRI, CT or TOE ${ }^{19}$. If stable, they recommend the use of MRI. Advantages of TOE as a diagnostic tool also extend to its versatility, and can be used throughout the hospital, from intensive care, emergency department and theatres to assess real time information regarding location and extent of dissection, which is ideal for patients who are haemodynamically unstable ${ }^{20}$. Dissection complications such as pericardial effusion and aortic insufficiency can be assessed $^{12}$. The disadvantages of TOE are of a practical nature; availability across hospital trusts, and it is an invasive procedure requiring an experienced technician due to operator-dependent results; sensitivity can vary, reported between 59 and $83 \%$, and specificity $63-93 \%^{12}$. With this knowledge, a high clinical suspicion should not deter from an additional investigation such as CT or MRI ${ }^{12}$.

\section{Aortopathies and Risk Stratification}


Women with pre-existing dilatation of the aorta, or disorders that make them more susceptible to dilatation, should be monitored throughout pregnancy as they have an increased relative risk for aortic complications ${ }^{3}$. It should be noted that many aortopathies are often only diagnosed following maternal death or cardiovascular event; in the Genetically Triggered Thoracic Aortic Aneurysms and Cardiovascular Conditions (GenTAC) study, $42 \%$ were aware of their MFS prior to their first pregnancy ${ }^{5,21}$. The aorta is counted as dilated once the diameter exceeds the upper limit of normal; 2 standard deviations greater than the mean predicted diameter matched for age and size ${ }^{22}$.

Women with known aortopathies should have a personalised plan for care during their pregnancy, including initial pre-conception counselling on their risk ${ }^{23}$. Heritable Thoracic Aortic Disease (HTAD), Loeys-Dietz syndrome, Vascular Ehlers-Danlos (vEDS), MFS, bicuspid aortic valve (BAV) and Turner syndrome (TS) are all known risk factors for developing $\mathrm{AAD}^{23}$. An entire imaging of the aorta should be concluded prior to pregnancy by CT or MRI, as well as exercise testing, ECG and echocardiography to assess risk ${ }^{5}$. Any aortic pathology in these risk groups warrant ongoing screening; for high-risk - those likely to suffer from $\mathrm{AD}$ or with already dilated aortas - echocardiography every 4 weeks is necessary, but for low-risk, every 12 weeks.

Counselling on vaginal or Caesarean delivery has been discussed depending on aortic diameter; vaginal delivery is deemed safe if the ascending aorta is less than $40 \mathrm{~mm}$, but Caesarean delivery should be considered in all those with ascending aorta $>40 \mathrm{~mm}$ but particularly in those $>45 \mathrm{~mm}$ or a prior history of $\mathrm{AD}^{5}$.

\section{Marfan Syndrome}

MFS is a heritable mutation in fibrillin-1 (FBN-1) affecting a number of organ systems including the predisposition to thoracic aortic aneurysms (TAA) and AAD. MFS affects approximately 1 in 5,000 people in the United States, and the highest association of AAD in pregnancy ${ }^{23,24}$. The increased risk of complications have been reported in numerous studies over the years with drastic consequences of AAD, but this information is debated to be skewed towards the complicated pregnancies ${ }^{24}$. In fact, the actual percentage risk of $\mathrm{AAD}$ in pregnancy with MFS is reported to be approximately $3-4.4 \%^{5,21,24}$.

If counselling is necessary for these patients, debate still exists on the nature of risk in MFS, with some suggestion that an aortic root below $40 \mathrm{~mm}$ has a relatively low risk of $1 \%^{5}$. The first study to show this was Pyeritz et al. in 1981, showing a low risk of complications and death when analysing 26 women with MFS across 105 pregnancies; their one maternal death had previous severe cardiac comorbidities, and they reported pregnancy in MFS as relatively safe for women without comorbidities ${ }^{25}$. A study by Rossiter et al . of 45 pregnancies in MFS noted that aortic diameters $<40 \mathrm{~mm}$ in MFS tolerated pregnancy well, and AD occurred in 2 patients with preceeding aortic risk ${ }^{26}$. Conversely, guidelines have recommended that MFS patients should not become pregnant if their ascending aorta diameter is greater than $45 \mathrm{~mm}$, or with severe comorbidities or family history of sudden death ${ }^{5}$. Aortic root diameter is influenced by sex, age and body surface area; assigning an arbitrary value of $40 \mathrm{~mm}$ and $45 \mathrm{~mm}$ may not be representative of every patient ${ }^{27}$.

For MFS patients with an aortic root $>40 \mathrm{~mm}$ but $<45 \mathrm{~mm}$, it is unclear how to accurately assess risk of AAD in pregnancy; a suggestion of risk estimates at $10 \%$ once $>40 \mathrm{~mm}^{22}$. Immer et al. recommended prepregnancy surgery with elective aortic root replacement in their study for those with aortic root dilatation $>40 \mathrm{~mm}^{28}$. Other studies have suggested intensive echocardiography monitoring every 4 to 8 weeks, with surgery considered in the event of rapid diameter growth. In one study, aortic diameter increased, on average, by $3 \mathrm{~mm}$ during pregnancy and did not fully recover at 5 -year follow up ${ }^{24}$. By this notion, multiple pregnancies become higher risk for women with MFS, and they remain at higher risk postpartum; it is not known how long for ${ }^{29}$. However, the growth rate was contested by two smaller studies, who found no difference in aortic root before and after pregnancy ${ }^{29}$. The jury on whether aortic root diameter changes during pregnancy is, as yet, undecided ${ }^{15}$.

\section{Bicuspid Aortic Valve}

BAV occurs in up to $2 \%$ of the population, rendering it the most common congenital cardiac malformation ${ }^{30}$. 
Investigating BAV may require other modalities than echocardiography, such as CT or MRI, due to the location of the dilatation (distal ascending aorta) ${ }^{5}$. BAV is regarded as low risk for dissection, at less than $1 \%^{5}$. Immer et al. found $10 \%$ of patients in their patients had BAV in their analysis of risk factors for AD during pregnancy; they were observed to be younger than patients with MFS (not significant), and dissections occurred earlier in pregnancy $(\mathrm{p}<0.05)^{28}$. Immer et al . warned that aortic root diameter in BAV $>40 \mathrm{~mm}$ was at risk of TAAD. The recommended requirement for elective aortic root surgery in BAV is $47 \mathrm{~mm}$ and above $^{5}$. A literature review by Yuan found 11 reports of AD in pregnant women with BAV ${ }^{30}$. 4 out of 11 women also had MFS or TS. The aortic diameter ranged between 29-70mm in these women, occurring even prior to reaching $40 \mathrm{~mm}$. Ascending aorta greater than $50 \mathrm{~mm}$ is recommended for women not to become pregnant ${ }^{5}$.

\section{Type IV Vascular Ehlers-Danlos}

vEDS is an autosomal dominant condition from mutations of COL3A $1^{31} .86 .7 \%$ of women were diagnosed with vEDS after completion of their pregnancy ${ }^{31}$. For patients with vEDS, pregnancy is contraindicated due to the high risks of dissection and uterine rupture ${ }^{5}$. However, this recommendation was based off a study that found there was no survival difference between nulliparous vEDS women and parous vEDS. Murrayet al . looked at maternal morbidity in their study of 565 pregnancies. Their mortality rate of pregnancy-related deaths was $5.3 \%$ (30). Arterial dissection or rupture occurred in 9.2\%, including 3 fatal aortic ruptures, 2 during labour at term, and 1 postpartum. Comparing Kaplan-Meier survival estimates of nulliparous versus parous vEDS and comparing groups of women not diagnosed due to complications and adjusting for mutation type, the mean age of death was approximately the same at 53 years $^{31}$. This led Murray et al . to conclude that the decision of pregnancy in vEDS should be based on expert discussion and planning with the patient involved, and not contraindicated based on genetic diagnosis. The use of celiprolol, a B1adrenoceptor antagonist, has also been noted to improve cardiovascular adverse events and could be utilised during pregnancy, subject to fetal monitoring ${ }^{22}$.

\section{Turner Syndrome}

One of the most common chromosomal abnormalities in women, TS occurs in 1 in 2500 live female births and is characterised complete or partial absence of the $\mathrm{X}$ chromosome ${ }^{32}$. Of these, up to $50 \%$ are expected to have cardiovascular malformations in addition to the common features of short stature and primary ovarian insufficiency ${ }^{33}$. Only $5-6 \%$ will have a spontaneous pregnancy ${ }^{34}$. For women with TS, oocyte donations have presented the opportunity for the majority to carry a pregnancy, but there is some debate whether the risk is too high ${ }^{34,35}$. Although TS itself is an increased relative risk of AD, estimated maternal mortality rates of $2 \%$ in TS from $\mathrm{AD}$ or rupture ${ }^{34}$. A study by Bernard et al. regarding outcomes in 480 patients found $5.6 \%$ had spontaneous pregnancies; none of these were associated with aortic root dilatation or aortic dissection ${ }^{36}$. Evidence shows that different karyotypes in TS may offer different risks; in Bernard's study, mosaic karyotype predisposed to spontaneous pregnancy, milder phenotype and therefore may have a lower risk to other karyotypes ${ }^{34,36}$. The recommendations from the 2016 Cincinnati International TS meeting suggested that full imaging, exercise testing and TTE should be performed 2 years prior to planned pregnancy or assisted reproductive therapy ${ }^{37}$. Any female with a prior history of AD should be advised against pregnancy, or if already pregnant, to be monitored closely and deliver by Caesarean. If there is no evidence of dilatation, TTE should be carried out at least once; suggested around 20 weeks, but if there is any evidence of dilatation, hypertension, BAV or other risk factors, monitored at least 1 to 2 monthly up to 6 months postpartum ${ }^{37}$. Using aortic size index (ASI) - absolute aortic diameter in cm divided by body surface area, which better predicts AD risk for TS - vaginal delivery is feasible at ASI less than $2 \mathrm{~cm}$. Above $2 \mathrm{~cm}$ and less than $2.5 \mathrm{~cm}$, Caesarean should be considered, but above $2.5 \mathrm{~cm}$ Caesarean is recommended ${ }^{37}$.

\section{Obstetric and Fetal Management}

\section{Pre-Conception}

Prior to conception, pre-pregnancy counselling, risk stratification and classification should guide management ${ }^{5,38}$. Ideally, surgical intervention prior to conception in at-risk women is indicated for cer- 
tain thresholds. 45mm ascending aorta diameter in MFS, FTAAD or SMAD3 mutation recommends repair prior to pregnancy; this is also the case of Loeys-Dietz $(40-45 \mathrm{~mm})$, BAV $(50 \mathrm{~mm})$, TS $(27 \mathrm{~mm}$, ASI) and others $(50 \mathrm{~mm})^{29}$.

\section{Medical management}

As noted earlier, many aortopathies are not known until pregnancy, and medical management, $\alpha$-Methyldopa or beta blocker can be utilised to slow aortic growth and advised by the $\mathrm{ESC}^{38}$. Gersonyet al . contested this, having found no difference between beta blocker use in a meta-analysis, and no change in dilatation ${ }^{39}$. In addition, beta blockade has been shown in several studies to have side effects, such as intrauterine growth restriction and fetal bradycardia ${ }^{15}$. A study of Celiprolol, however, had a decrease of arterial events (20\%) in a randomised control trial compared to control (50\%), but this has not been demonstrated in patients with aortic disease ${ }^{15}$.

\section{Surgical management}

The consideration when operating is weighing up the risk of delivering the fetus concurrently with aortic repair for TAAD or operating with fetus in utero . Yates et al reported their series of 11 patients who had emergency cardiac surgery requiring urgent delivery of the fetus ${ }^{40}$. They succeeded with no maternal deaths but reported a fetal mortality rate of $27 \%$. The precise timing of when to deliver varies around the age of the fetus, with some authors such as Zeebregts suggesting between 28 - 32 weeks the option for concomitant delivery should fetal maturity have occurred ${ }^{8,9,41}$. Others, such as the European Society of Cardiology, recommend delivery from 26 weeks gestation, when fetal maturity is likely to have been reached ${ }^{8}$. In a systematic review by de Martinoet al, analysis of seven studies and 63 patients represented a maternal and fetal mortality of 23 and $27 \%$ respectively ${ }^{42}$. Whilst concerning, they questioned whether the maternal mortality was similar in non-pregnant women, as suggested by a longitudinal observational cohort study.

\section{Aortic repair with fetus in utero}

If delivery of the fetus is not possible - in general, this is at less than 28 weeks - there are limited studies and evidence regarding CPB and pregnancy, bar the consensus that it is a high-risk, with high fetal and maternal mortality ${ }^{43}$. Fetal mortality is a significant risk during CPB, with estimates of around $3-20 \%$, with highest risk in early gestational age ${ }^{8,40}$. If the decision to perform aortic surgery with the foetus in-utero is made, it is necessary to take steps to maximise chances of foetal survival. Therefore, intra-operative conditions must be optimised to facilitate this, the focus of which will be maintaining placental blood flow and foetal circulation, and greater studies needed to focus on which strategies are best.

Foetuses have limited ability to modify their stroke volume thus cardiac output is highly dependent on heart rate $^{44}$. Consequently, it is important to minimise foetal bradycardia. Foetal bradycardia has long been associated with poor placental perfusion as described by Koh et $\mathrm{al}^{45}$. Early case studies have also reported resolution of foetal bradycardia on increasing flow rate $\mathrm{e}^{46-48}$. This lead to some authors recommending that CPB flow rate should be maintained over $2.5 \mathrm{~L} / \mathrm{min} / \mathrm{m}^{2}$, with a MAP of $70-75 \mathrm{mmHg}^{49}$.

Haemodilution should be minimised, and there should be an intra-operative target of a haematocrit above $25 \%^{50}$. The proposed benefit is twofold; a high haematocrit increases the oxygen carrying capacity of the blood, and delivery to the foetus, whilst preventing significant reduction in the concentration of circulating progesterone which can trigger uterine contractions and disrupt placental blood flow ${ }^{9}$. Experimental evidence suggests that use of pulsatile flow should be used when available as this can increase uterine and placental perfusion by stimulating nitric oxide production and local vasodilatation ${ }^{51}$.

Analysis of case studies has shown a link between hypothermia during CPB and increased foetal mortality ${ }^{4,8}$. One hypothesis for this increase in mortality is due to induced foetal bradycardia. Hypothermia can also induce uterine contractions and labours, particularly in the rewarming phase ${ }^{50}$. Therefore, many authors recommend avoiding temperatures below 35 degrees. However there have been case reports of successful deep hypothermic circulatory arrest performed with foetus in-utero, and some bigger retrospective studies ${ }^{8,28,41,52,53}$. Normothermic perfusion was recommended by Becker in 1983, or antegrade cerebral perfusion with moderate 
hypothermia, used by Bachet and Guilmet; no single technique has been proven to greater efficacy than the other $^{28}$.

\section{Postpartum management}

Until recently, a hysterectomy was routinely recommended due to the risk of postpartum haemorrhage. However, use of a Cook balloon has been noted by several studies to have been effective at preventing this ${ }^{1,8}$.

\section{Conclusions}

The challenges faced when dealing with AD in pregnancy are numerous, and for best outcomes an individual approach is needed. Diagnosis must be rapid, and management initiated in order to save both mother and baby. Despite this, challenges carrying out management in the form of operative techniques and cardiopulmonary bypass place the foetus at risk and must be approached with caution, particularly as there is little evidence-base for many of these decisions. Further research into reducing maternal and fetal mortality is necessary.

References

1. Zhu JM, Ma WG, Peterss S, et al. Aortic Dissection in Pregnancy: Management Strategy and Outcomes. Ann Thorac Surg . 2017;103(4):1199-1206. doi:10.1016/j.athoracsur.2016.08.089

2. Mittauer E, Harris K, Evangelista-masip A, et al. AORTIC DISSECTION RELATED TO PREGNANCY: THE INTERNATIONAL REGISTRY OF ACUTE AORTIC DISSECTION (IRAD). J Am Coll Cardiol . 2020;75(11):2213. doi:10.1016/S0735-1097(20)32840-0

3. Kamel H, Roman M, Pitcher A, Deverueux R. Pregnancy and the Risk of Aortic Dissection or Rupture: a Cohort-Crossover Analysis.Circulation . 2016;134(7):527-533. doi:10.1016/j.physbeh.2017.03.040

4. Patel PA, Fernando RJ, MacKay EJ, et al. Acute Type A Aortic Dissection in Pregnancy—Diagnostic and Therapeutic Challenges in a Multidisciplinary Setting. J Cardiothorac Vasc Anesth . 2018;32(4):1991-1997. doi:10.1053/j.jvca.2018.01.035

5. Regitz-Zagrosek V, Roos-Hesselink JW, Bauersachs J, et al. 2018 ESC Guidelines for the Management of Cardiovascular Diseases during Pregnancy . Vol 39.; 2018. doi:10.1093/eurheartj/ehy340

6. Sun HD, Hsiao SM, Yang ST, Cheng NM, Wang PH. Aortic dissection in the third trimester pregnancy without risk factors. Taiwan J Obstet Gynecol . 2019;58(5):723-724. doi:10.1016/j.tjog.2019.07.029

7. Aziz F, Penupolu S, Alok A, Doddi S, Abed M. Peripartum acute aortic dissection: A case report \& review of literature. J Thorac Dis . 2011;3(1):65-67. doi:10.3978/j.issn.2072-1439.2010.11.12

8. Chen SW, Zhong YL, Ge YP, et al. Successful repair of acute type A aortic dissection during pregnancy at 16th gestational week with maternal and fetal survival: A case report and review of the literature. World J Clin Cases . 2019;7(18):2843-2850. doi:10.12998/wjcc.v7.i18.2843

9. Zeebregts CJ, Schepens MA, Hameeteman TM, Morshuis WJ, De La Rivière AB. Acute aortic dissection complicating pregnancy. Ann Thorac Surg . 1997;64(5):1345-1348. doi:10.1016/S0003-4975(97)00916-8

10. Thalmann M, Sodeck GH, Domanovits H, et al. Acute type A aortic dissection and pregnancy: A population-based study. Eur J Cardio-thoracic Surg . 2011;39(6):e159-e163. doi:10.1016/j.ejcts.2010.12.070

11. Yam N, Lo CSY, Ho CKL. Acute aortic dissection associated with pregnancy. Ann Thorac Surg . 2015;100(4):1470. doi:10.1016/j.athoracsur.2015.07.008

12. Ch'ng SL, Cochrane AD, Goldstein J, Smith JA. Stanford Type A Aortic Dissection in Pregnancy: A Diagnostic and Management Challenge.Hear Lung Circ . 2013;22(1):12-18. doi:10.1016/j.hlc.2012.08.005

13. Abo-Salem E, López-Candales A. Diagnosis of asymptomatic aortic dissection during pregnancy using contrast echocardiography. J Cardiol Cases . 2014;9(5):200-202. doi:10.1016/j.jccase.2014.01.007 
14. Lameijer H, Crombach A. Aortic Dissection During Pregnancy or in the Postpartum Period: It All Starts With Clinical Recognition. Ann Thorac Surg . 2018;105(2):663. doi:10.1016/j.athoracsur.2017.05.054

15. Van Hagen IM, Roos-Hesselink JW. Aorta pathology and pregnancy.Best Pract Res Clin Obstet Gynaecol . 2014;28(4):537-550. doi:10.1016/j.bpobgyn.2014.03.007

16. Clough RE, Nienaber CA. Management of acute aortic syndrome.Nat Rev Cardiol . 2015. doi:10.1038/nrcardio.2014.203

17. Committee opinion no. 723: guidelines for diagnostic imaging during pregnancy and lactation. The American College of Obstetricians and Gynecologists. Obstet Gynecol . 2017;130(4):210-216.

18. Lowe S. Diagnostic radiography in pregnancy: risks and reality. Aust N Z L Obs Gynaecol . 2004;44(3):191196.

19. Erbel R, Aboyans V, Boileau C, et al. 2014 ESC Guidelines on the diagnosis and treatment of aortic diseases: Document covering acute and chronic aortic diseases of the thoracic and abdominal aorta of the adult. Eur Heart J . 2014. doi:10.1093/eurheartj/ehu281

20. Evangelista A, Flachskampf FA, Erbel R, et al. Echocardiography in aortic diseases: EAE recommendations for clinical practice. Eur J Echocardiogr . 2010. doi:10.1093/ejechocard/jeq056

21. Roman MJ, Pugh NL, Hendershot TP, et al. Aortic Complications Associated With Pregnancy in Marfan Syndrome: The NHLBI National Registry of Genetically Triggered Thoracic Aortic Aneurysms and Cardiovascular Conditions (GenTAC). J Am Heart Assoc . 2016;5(8):1-7. doi:10.1161/JAHA.116.004052

22. Muiño Mosquera L, De Backer J. Managing aortic aneurysms and dissections during pregnancy. Expert Rev Cardiovasc Ther . 2015;13(6):703-714. doi:10.1586/14779072.2015.1042862

23. Rajagopalan S, Nwazota N, Chandrasekhar S. Outcomes in pregnant women with acute aortic dissections: A review of the literature from 2003 to 2013. Int J Obstet Anesth . 2014;23(4):348-356. doi:10.1016/j.ijoa.2014.05.001

24. Goland S, Elkayam U. Pregnancy and Marfan Syndrome. Ann Cardiothorac Surg . 2017;6(6):642-653.

25. Pyeritz R. Maternal and fetal complications of pregnancy in the Marfan syndrome. Am J Med . 1981;71:784-790.

26. Rossiter JP, Repke JT, Morales AJ, Murphy EA, Pyeritz RE. A prospective longitudinal evaluation of pregnancy in the Marfan syndrome.Am J Obstet Gynecol . 1995;173(5):1599-1606. doi:10.1016/00029378(95)90655-X

27. Vriz O, Aboyans V, D'Andrea A, et al. Normal values of aortic root dimensions in healthy adults. Am $J$ Cardiol . 2015;114(6):921-927. doi:10.1016/j.amjcard.2014.06.028

28. Immer FF, Bansi AG, Immer-Bansi AS, et al. Aortic dissection in pregnancy: Analysis of risk factors and outcome. Ann Thorac Surg . 2003;76(1):309-314. doi:10.1016/S0003-4975(03)00169-3

29. Wanga S, Silversides C, Dore A, de Waard V, Mulder B. Pregnancy and thoracic aortic disease: Managing the risks. Can J Cardiol . 2016;32(1):78-85. doi:10.1016/j.cjca.2015.09.003

30. Yuan SM. Bicuspid aortic valve in pregnancy. Taiwan J Obstet Gynecol . 2014;53(4):476-480. doi:10.1016/j.tjog.2013.06.018

31. Murray ML, Pepin M, Peterson S, Byers PH. Pregnancy-related deaths and complications in women with vascular Ehlers-Danlos syndrome. Genet Med . 2014;16(12):874-880. doi:10.1038/gim.2014.53

32. Thunström S, Krantz E, Thunström E, Hanson C, Bryman I, Landin-Wilhelmsen K. Incidence of Aortic Dissection in Turner Syndrome: A 23-Year Prospective Cohort Study. Circulation . 2019;139:2802-2804. 
33. Karnis MF, Zimon AE, Lalwani SI, Timmreck LS, Klipstein S, Reindollar RH. Risk of death in pregnancy achieved through oocyte donation in patients with Turner syndrome: A national survey.Fertil Steril . 2003;80(3):498-501. doi:10.1016/S0015-0282(03)00974-9

34. Söderström-Anttila V, Pinborg A, Karnis MF, Reindollar RH, Paulson RJ. Should women with Turner syndrome be allowed to carry their own pregnancies? Fertil Steril . 2019;112(2):220-225. doi:10.1016/j.fertnstert.2019.06.003

35. Paulson RJ. Pregnancies in women with Turner Syndrome: how do we counsel our patients? Fertil Steril . 2019;112(2):219. doi:10.1016/j.fertnstert.2019.06.001

36. Bernard V, Donadille B, Zenaty D, et al. Spontaneous fertility and pregnancy outcomes amongst 480 women with Turner syndrome. Hum Reprod . 2016;31(4):782-788. doi:10.1093/humrep/dew012

37. Gravholt CH, Andersen NH, Conway GS, et al. Clinical practice guidelines for the care of girls and women with Turner syndrome: Proceedings from the 2016 Cincinnati International Turner Syndrome Meeting. Eur $J$ Endocrinol . 2017;177(3):G1-G70. doi:10.1530/EJE-17-0430

38. Bons LR, Roos-Hesselink JW. Aortic disease and pregnancy. Curr Opin Cardiol . 2016;31(6):611-617. doi:10.1097/HCO.0000000000000336

39. Gersony DR, McClaughlin MA, Jin Z, Gersony WM. The effect of beta-blocker therapy on clinical outcome in patients with Marfan's syndrome: A meta-analysis. Int J Cardiol . 2007;114(3):303-308. doi:10.1016/j.ijcard.2005.11.116

40. Yates MT, Soppa G, Smelt J, et al. Perioperative management and outcomes of aortic surgery during pregnancy. J Thorac Cardiovasc Surg . 2015;149(2):607-610. doi:10.1016/j.jtcvs.2014.10.038

41. Sakaguchi M, Kitahara H, Seto T, et al. Surgery for acute type A aortic dissection in pregnant patients with Marfan syndrome. Eur J Cardio-thoracic Surg . 2005;28(2):280-283. doi:10.1016/j.ejcts.2005.02.047

42. De Martino A, Morganti R, Falcetta G, et al. Acute aortic dissection and pregnancy: Review and meta-analysis of incidence, presentation, and pathologic substrates. J Card Surg . 2019;34(12):1591-1597. doi:10.1111/jocs.14305

43. Saito A. Editorial: Pregnant patients and open-heart surgery - Decision-making for appropriate timing and surgical strategy. J Cardiol Cases . 2016;13(3):70-71. doi:10.1016/j.jccase.2015.12.006

44. Thompson LP, Hashimoto K. The Fetal Circulation. Anesth Fetus . 2012;5(4):32-38. doi:10.1002/9781118477076.ch4

45. Koh KS, Friesen RM, Livingstone RA, Peddle LJ. Fetal monitoring during maternal cardiac surgery with cardiopulmonary bypass. Obstet Gynecol Surv . 1975;30(11):739-740. doi:10.1097/00006254-197511000-00010

46. Werch A, Michael Lambert H, Cooley D, Reed CC. Fetal monitoring and maternal open heart surgery. South Med J . 1977;70(8):1024. doi:10.1097/00007611-197708000-00045

47. Levy D, Warriner R. Fetal response to cardiopulmonary bypass. Obs Gynecol . 1980;56(1):112-115.

48. Eilen B, Kaiser IH, Becker RM, Cohen MN. Aortic valve replacement in the third trimester of pregnancy: Case report and review of the literature. Obstet Gynecol . 1981;57(1):119-121. doi:10.1097/00132582198203000-00004

49. Parry AJ, Westaby S. Cardiopulmonary bypass during pregnancy.Ann Thorac Surg . 1996;61(6):18651869. doi:10.1016/0003-4975(96)00150-6

50. Yuan S-M. Indications for Cardiopulmonary Bypass During Pregnancy and Impact on Fetal Outcomes. Geburtshilfe Frauenheilkd . 2014;74(01):55-62. doi:10.1055/s-0033-1350997 
51. Vedrinne C, Tronc F, Martinot S, et al. Better preservation of endothelial function and decreased activation of the fetal renin-angiotensin pathway with the use of pulsatile flow during experimental fetal bypass. J Thorac Cardiovasc Surg . 2000;120(4):770-777. doi:10.1067/mtc.2000.108902

52. Sepehripour AH, Lo TT, Shipolini AR, McCormack DJ. Can pregnant women be safely placed on cardiopulmonary bypass? Interact Cardiovasc Thorac Surg . 2012;15(6):1063-1071. doi:10.1093/icvts/ivs318

53. Plunkett MD, Bond LM, Geiss DM. Staged repair of acute type I aortic dissection and coarctation in pregnancy. Ann Thorac Surg . 2000;69(6):1945-1947. doi:10.1016/S0003-4975(00)01278-9 